\title{
Modelando acessibilidade na web: uma proposta para o desenvolvimento de material didático baseado no perfil do usuário
}

\author{
Patrícia B. Scherer Bassani, Feevale, patriciab@feevale.br \\ Marine Bello Flores, Feevale, marine@ feevale.br \\ Marcelo Ritzel, Feevale, ritzel@feevale.br \\ Regina de Oliveira Heidrich, Feevale, rheidrich@feevale.br
}

Resumo: Este estudo se insere nas pesquisas que vêm sendo realizadas na área de educação a distância, enfocando o desenvolvimento de material instrucional para cursos Web. Busca articular as pesquisas nas áreas de Inteligência Artificial e Web Semântica, de forma a propor uma ontologia que oriente a ação de um agente capaz de perceber as interações entre usuário e o ambiente, de forma a identificar o perfil do aluno a distância, especialmente no que se refere a navegabilidade, interface e cognição, ou mesmo o conhecimento sobre a interface. $\mathrm{O}$ modelo proposto busca contemplar sujeitos com diferentes necessidades visuais, auditivas e cognitivas.

\section{Palavras-Chave}

educação a distância, ontologias, agentes, acessibilidade

\section{Modeling accessibility in web: a proposal for the development of instrucional material based in user profile}

\begin{abstract}
This study contributes to the research that has been taken in distance learning study area, focusing the development of instructional content for Web courses. The article has the goal to articulate the research in Artificial Intelligence and Web Semantics areas to consider an ontology to guide the action of an agent capable to perceive the interactions between user and the environment. The objective of this agent is to identify the profile of the distance student, especially with respect to navigability, interface and cognition. The model try to contemplate users with different visual, auditory and cognitives necessities.
\end{abstract}

\section{Keywords}

distance learning, ontology, agents, accessibility

\section{INTRODUÇÃ̃o}

Diferentes tecnologias incorporadas ao processo de ensino-aprendizagem foram responsáveis pela forma de elaboração dos materiais dos cursos na modalidade a distância. O início (primeira geração) foi marcado pela utilização de material impresso, como livros, cartilhas e guias de estudo; a seguir (segunda geração), televisão, rádio, áudio e vídeo foram incorporados ao ensino; a partir dos anos 90 (terceira geração), o desenvolvimento e expansão da tecnologia de redes de comunicação e o acesso à Internet aumentaram as possibilidades de comunicação bidirecional, desvelando novas formas de interação, por meio de recursos de teleconferência e/ou videoconferência, consulta a bancos de dados remotos e facilidade de acesso a conteúdos (Peters, 2001, Litwin, 2001).

As técnicas de Inteligência Artificial (IA) têm se constituído em objeto de crescente investigação por parte dos pesquisadores da área de informática aplicada à educação, devido, principalmente, às suas potencialidades, no que se refere ao desenvolvimento de ambientes de ensino-aprendizagem inteligentes. 
Ambientes de ensino-aprendizagem inteligentes são sistemas que, na interação com o aluno, modificam suas bases de conhecimento através das percepções feitas. Possuem a capacidade de aprender e adaptar estratégias de ensino de acordo com o desempenho do aluno, e se caracterizam, sobretudo, por construir um Modelo Cognitivo desse aluno, através da interação, da formulação e da comprovação de hipóteses sobre seu conhecimento. Possui, contudo, a capacidade de adequar estratégias de ensinoaprendizagem ao aluno e à situação atual (Viccari, 1990). Sendo assim, estes ambientes computacionais têm um grande potencial para uso pedagógico, uma vez que envolve o usuário/aluno no processo, potencializando alto grau de interação.

Por outro lado, entende-se que os estudos na área de Web Semântica, especialmente no que se refere a ontologias, apresentam uma nova proposta de modelagem, que busca representar e descrever a realidade de um determinado domínio do conhecimento. As ontologias são consideradas um componente fundamental na Web Semântica, por promoverem padrões de estruturação e definição de significado dos termos de um domínio do conhecimento e ainda gerarem um meio para descrever a semântica de documentos Web, permitindo às aplicações e aos agentes inteligentes fazerem uso dessa semântica (Novello, 2002).

Nesta perspectiva, este estudo busca uma articulação entre as pesquisas nas áreas de Inteligência Artificial e Web Semântica, de forma a propor uma ontologia, criando uma rede de conhecimentos, que oriente a ação de um agente capaz de perceber as interações entre usuário e ambiente, de forma a identificar o perfil do aluno a distância. Este perfil envolve aspectos relacionados à navegabilidade, interface e cognição, de forma a contemplar sujeitos com diferentes necessidades visuais, auditivas e cognitivas. Este artigo apresenta os resultados parciais de um estudo em desenvolvimento e busca contribuir com pontos referenciais para o desenvolvimento de material instrucional adaptativo para cursos a distância.

\section{WEB SEMÂNTICA E ONTOLOGIAS}

Berners-Lee et al. (2001), propõe uma Web em que a informação seja filtrada não só por humanos, mas também pelas máquinas; uma Web que seja uma extensão da Web atual, a chamada Web Semântica, onde as informações estejam organizadas de forma que as máquinas possam, de maneira inteligente, integrar recursos.

Atualmente, a Web está organizada com recursos que não facilitam a busca de informações, deixando para humanos a tarefa de filtrá-las. Para melhor filtrar as informações, o W3C (World Wide Web Consortium), prevê um conjunto de padrões que permite, às aplicações, trocarem informações de forma automatizada e inteligente.

Estes padrões podem ser representados em camadas, em formato de "bolo de noiva", onforme apresentando na igura 1 , onde cada camada subseqüente estende a funcionalidade e a expressividade das camadas anteriores. Na sequência, as três primeiras camadas são compostas pela URI (Uniform Resource Identifiers) e Unicode, XML (Extensible Markup Language), Namespaces e XML Schema. Estas camadas são consideradas fundamentais na estruturação de documentos, pois são nestas camadas que são definidas a forma como a informação deve ser apresentada.

As camadas seguintes, RDF (Resource Description Framework), RDF Schema e Ontology, informam o significado da informação, ou seja a semântica de um dado domínio do conhecimento bem como seus axiomas. A camada de ontologia serve para estabelecer um vocabulário comum e compartilhado, sendo possível trocar informações entre os objetos. 


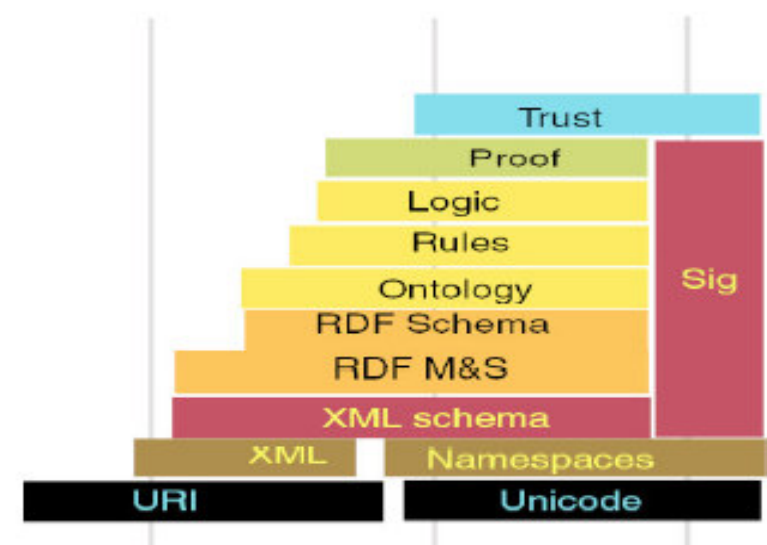

Figura 1: Linguagens da Web Semantica (fonte: http://www.w3.org/2001.sw)

O termo ontologia advindo da filosofia e utilizado por Aristóteles para designar o estudo do ser enquanto ser, é sugerido por Sowa (2000), para categorizar o que existe ou pode existir num determinado domínio do conhecimento.

Atualmente, o termo ontologia vem sendo empregado largamente em Ciência da Computação e, segundo sua aplicabilidade, sua definição assume algumas variantes. Em Inteligencia Artificial (IA) ele foi apresentado pela primeira vez por Neches et al. (2001): "An ontology defines the basic terms and relations comprising the vocabulary of a topic area, as well as the rules for combining terms and relations to define extensions to the vocabulary". A definição de ontologia mais conhecida foi aquela dada por Gruber (1993): "An ontology is an explicit specification of a conceptualization". Já Uschold e Gruninger (1996), a definem da seguinte maneira: "An ontology is a shared understanding of some domain of interest".

Maedche e Staab (2001), nos apresentam uma conceituação de ontologia mais formal, expressa da seguinte forma: $\mathrm{O}=\{\mathrm{C}, \mathrm{R}, \mathrm{I}(\mathrm{C}), \mathrm{I}(\mathrm{R}), \mathrm{A}\}$, onde $\mathrm{C}$ e $\mathrm{R}$ são dois conjuntos disjuntos, em que o $\mathrm{C}$ é utilizado para representar o conjunto de conceitos/classes de um determinado domínio e o R representa um conjunto de relações entre estes conceitos; o I(C) representa um conjunto de instâncias dos conceitos em $\mathrm{C}$ e o I $(\mathrm{R})$ representa um conjunto de relações para cada relação em $\mathrm{R}$; o A representa o conjunto de Axiomas expressos em linguagem lógica apropriada referentes aos conceitos e as relações definidas em $\mathrm{O}$.

Gómez-Pérez et al. (2004), definem ontologias como sendo constituídas de conceitos/classes, que são definidos por um conjunto de atributos e seus possíveis valores; relações, que representam os tipos de associações entre elementos do domínio; funções, que são tipos especiais de relações que mapeiam um ou mais elementos do domínio para um elemento único; axiomas, sentenças sobre o domínio que são sempre verdadeiras e que restringem as interpretações e o uso de nomes e instâncias, que são consideradas os indivíduos da ontologia.

Generalizando, pode-se dizer que as ontologias constituiem-se de conceitos que associam nomes de entidades representando o conhecimento declarativo de um domínio. Assim, as ontologias podem ser utilizadas como uma teoria lógica de um domínio de conhecimento.

Complementando o conjunto de padrões sugeridos pelo W3C, na estrutura da Web Semântica, tem-se ainda as camadas de regras e lógica que provêem os formalismos, a fim de permitir o processamento automático das informações, permitindo o raciocínio de máquinas, seguida da camada de prova. A última camada se caracteriza por ser a camada da confiança, onde as assinaturas digitais funcionam como mecanismos com a finalidade de evitar inconsistências, servindo para garantir a segurança das informações. 
Por sua vez, o modelo de Web Semântica, previsto por Berners-Lee et al. (2001), é fundamentado em ontologias, agentes de softwares semânticos e assinaturas digitais.

As linguagens de representação de ontologias são diversas, entre elas destaca-se a OWL (Web Ontology Language), oriunda da linguagem DAML + OIL. OWL permite descrever ontologias segundo as normas definidas pelo W3C, possibilitando a definição de um conjunto de classes, relacionamentos e restrições de interpretação por meio de axiomas. A OWL caracteriza-se por sua robustez ao possuir mais facilidades para expressar significados e semânticas do que XML, RDF e RDF Schema, embora seja baseada em RDF e RDF Schema e utilize a sintaxe XML. A OWL é ideal no uso de aplicações que necessitem processar o conteúdo de informações, ao invés de somente apresentar a visualização destas informações. A OWL é dividida em três sublinguagens:

a) OWL Lite, que se caracteriza por ser uma linguagem que dá suporte aos usuários que necessitam de uma hierarquia de classificação e funcionalidades com restrições simples.

Ela possui algumas limitações nos recursos oferecidos, entre eles o uso de cardinalidade, que apesar de estar disponível é restrita a obter somente os valores 0 ou 1. A OWL Lite torna-se mais fácil de ser implementada e faz com que a transição de outros modelos de vocabulários e taxonomias para OWL seja mais rápida (OWL, 2004);

b) OWL-DL é uma linguagem que dá suporte aos usuários que necessitam de um pouco mais de expressividade na formalização de ontologias (OWL, 2004). A OWL-DL além de oferecer todos os recursos apresentados pela OWL Lite, oferece também outros mecanismos de linguagem, como impor algumas restrições quanto ao uso de recursos tais como, uma classe não ser instância e propriedade ao mesmo tempo (OWL, 2004);

c) OWL Full é a mais expressiva, pois além de oferecer todos os vocabulários disponíveis pela linguagem OWL, não impõe restrição ao uso dos recursos (OWL, 2004), não impondo restrição aos valores das classes, propriedades, instâncias e dados (OWL, 2004). Na OWL Full existe a possibilidade de uma classe ser tratada ao mesmo tempo como instância de classe.

A camada de ontologias, na Web Semântica, tem como finalidade fornecer um padrão bem definido e estruturado da informação. As ontologias, por serem um conjunto de vocábulos estruturados que permitem explicar a relação entre os diferentes termos evitando ambigüidades, são utilizadas na representação de conhecimento. Desta forma, permitem consultas baseadas em conceitos e relacionamentos de um determinado domínio do conhecimento.

Neste trabalho, utiliza-se a OWL-DL, uma vez que é suficiente para o domínio a ser formalizado e, principalmente, por impor restrições quanto ao uso de recursos.

\subsection{Modelando a acessibilidade na Web}

O Design Universal (Universal Design), também conhecido como Design para Todos, orienta o design de produtos e de ambientes, de forma que estes possam ser utilizados pela maioria das pessoas, sem a necessidade de adaptação ou design especializado.

Dos sete (7) princípios básicos do Design Universal, entende-se que alguns devem ser contemplados no desenvolvimento de softwares/ambientes educacionais, que são:

a) uso equiitativo: refere-se ao fato do software ser útil e poder ser utilizado por pessoas com diversas capacidades. Deve-se verificar se o software proporciona a mesma forma de utilização a todos os usuários (idêntica sempre que possível; equivalente se necessário) e se ele e evita segregar ou estigmatizar quaisquer usuários;

b) flexibilidade no uso: envolve a possibilidade de escolher a forma da utilização, como, por exemplo, acesso e uso ao destro ou canhoto, e adaptabilidade ao ritmo do usuário;

c) uso simples e intuitivo: o software deve apresentar facilidade de uso e fácil compreensão, independentemente da experiência, do conhecimento, das capacidades lingüísticas ou do atual nível de concentração do usuário. Sendo assim, torna-se importante eliminar a 
complexidade desnecessária, sendo coerente com as expectativas e a intuição do usuário, acomodando um amplo leque de capacidades linguiísticas e níveis de instrução, além da informação estar organizada de forma coerente com a sua importância;

d) informação perceptível: aborda a questão da comunicação eficaz ao usuário, apresentando a informação necessária, independentemente das suas capacidades sensoriais ou das condições ambientais. Para tanto, pode envolver diferentes modos para apresentar informação essencial (pictográfico, verbal, táctil); maximiza a "legibilidade" de informação essencial, diferencia os elementos em formas que possam ser descritas (fazer com que seja fácil dar instruções ou orientações) e é compatível com a diversidade de técnicas ou equipamentos utilizados por pessoas com limitações sensoriais.

Os estudos e normatizações da W3C apontam, que a avaliação da acessibilidade deve perpassar todo o projeto de desenvolvimento de um ambiente computacional. A implementação de recursos de acessibilidade em ambientes colaborativos na Web ampliam as possibilidades de "acesso à informação e a interação de pessoas portadoras de necessidades especiais de forma inédita". Os estudos do W3C sugerem que, "ao desenvolver ou redesenhar um site, a avaliação da acessibilidade logo no início e ao longo do trabalho detectará itens de acessibilidade que se tornarão mais fáceis de corrigir do que se deixarmos o trabalho de avaliação para o final do projeto".

Nesta perspectiva, entende-se que a proposição de uma ontologia que oriente a ação de um agente capaz de perceber as interações que se constituem entre sujeito-usuário e o meio, constitui importante elemento para identificar o perfil do aluno usuário e possibilita novos referenciais para o desenvolvimento de material instrucional adaptativo de forma a contemplar sujeitos com diferentes necessidades físicas (por exemplo: tamanho de letra, leitor de tela) e/ou cognitivas.

\section{AGENTES}

Entende-se que um agente é uma entidade que trabalha de forma autônoma e sem interrupção em um sistema/ambiente. Segundo Ferreira e Bercht (2000), "agente é uma entidade real ou virtual, inserida em um ambiente sobre onde é capaz de atuar, dispondo de capacidade de percepção e de representação parcial deste ambiente, podendo se comunicar com outros agentes e possuindo um comportamento autônomo, conseqüência de suas observações, de seu conhecimento e de suas intenções com outros agentes. Um agente raciocina sobre o ambiente, sobre os outros agentes e decide, racionalmente, qual objetivo deve perseguir e qual ação deve tomar".

Um agente deve representar as seguintes características: Autonomia: operar sem intervenção direta de humanos, e tem controle sobre suas ações e seu próprio estado interno; Mobilidade: locomoção para outros ambientes; Continuidade temporal: funcionar continuamente; Adaptabilidade; e Comportamento ou habilidade social: interagir com outros agentes ou humanos.

Entretanto, é importante mencionar outras características ditas mais complexas, como:

Reatividade (percepção): perceber o ambiente onde está, detectando alterações, e responder em tempo hábil as mudanças nesse ambiente; Pró-atividade: comportamento encaminhado à objetivos, tomando a iniciativa de ação para cumprir suas metas; Emoção; Processamento de linguagem natural.

É importante que se tenha em mente que não é possível representar o conhecimento de modo completo e imutável, o que leva a necessidade de se capacitar os agentes com habilidade de aprender.

\subsection{Arquitetura}

Para Jennings (1998) "uma arquitetura é um mapa interno de um agente: suas estruturas de dados, as operações que podem ser executadas e ofluxo de controle destes". 
Alguns autores (Wooldridge, 1997), dividem tradicionalmente em três gêneros: agentes deliberativos ou cognitivos, agentes reativos e agentes híbridos.

Os agentes deliberativos utilizam a abordagem simbólica da IA e possuem como componentes básicos um modelo simbólico do ambiente, uma especificação simbólica das ações disponíveis e um algoritmo de planejamento. Um agente tem cognição quando possui uma representação simbólica do mundo e toma suas decisões considerando essa representação. Assim, esses agentes são capazes de raciocinar a respeito de suas intenções e conhecimentos, criar planos de ação e executá-los. Possuem modelos explícitos do mundo externo, estruturas de memória que o permitem manter um histórico de ações passadas e fazer previsões de ações futuras, e um sistema desenvolvido de cooperação e comunicação.

Os agentes reativos baseiam-se em uma hierarquia reativa, onde os agentes são projetados como um conjunto de tarefas executadas de forma comportamental. Apesar de simples isoladamente, os agentes reativos têm sua força no trabalho em grupo, o que possibilita que realizem tarefas impossíveis individualmente (Alvares, 1997). Eles não usam raciocínio simbólico complexo, estruturas de memória e uma representação interna explícita do conhecimento. Assim, não possuem um histórico de ações passadas, e não pode haver previsões de atos futuros. Esse agente pode, apenas, perceber o ambiente externo e, baseado nos estímulos recebidos, reagir de forma predeterminada.

Os agentes híbridos fazem parte de uma arquitetura mais recente, que busca conciliar as técnicas empregadas nos reativos e nos deliberativos.

De uma forma geral, os agentes reativos, possuem uma base (tabela) de referência para responder às ações do sistema, executando-as de forma predeterminada, e os agentes cognitivos/deliberativos, são vistos como sistemas de intenção, ou seja, possuem estados mentais de informação e manipulam o conhecimento. Entende-se como estados mentais: crença, desejo, conhecimento, intenção, etc., que são representados internamente nos agentes.

Os agentes podem ser hierarquizados de acordo com a principal atividade que desempenham no sistema. Algumas das denominações mais comuns para os agentes são agentes colaborativos, agentes de interface, agentes assistentes, agentes de recuperação de informação, e outros.

Pode-se notar que, durante o desenrolar das ações em um ambiente computacional voltado ao ensino, tanto o usuário quanto os outros agentes, devem estar cientes das mudanças no espaço do ambiente ao seu redor, o que caracteriza sua acessibilidade.

Os agentes, em geral, têm um conjunto de ações que podem ser executadas para modificar o ambiente. A questão chave é decidir qual ação executar para alcançar os objetivos planejados, dadas determinadas pré-condições. No caso de um agente puramente reativo, as suas ações são determinadas unicamente pelo estado atual do ambiente. Outros tipos de agente baseiam-se em seu próprio estado interno, além das impressões do ambiente para a tomada de decisões.

Esta pesquisa envolve o desenvolvimento de um agente do tipo deliberativo. Sua base de conhecimentos tem origem a partir da ontologia proposta.

No entanto, a ontologia proposta parte de uma base conceitual elaborada a partir de estudos teóricos, para ser ampliada a medida que o agente atua no ambiente, percebendo novas possibilidades de interação.

Nesta perspectiva, as interações percebidas a partir dos agentes são causa e consequiência, buscam na ontologia orientação para suas decisões, bem como as alimentam com percepções feitas a partir das interações.

\section{MAPEAMENTO DAS INTERAÇÕES}

A interação pode ser abordada a partir de uma perspectiva epistemológica ou tecnológica. A interação, numa abordagem epistemológica, estuda as relações entre o desenvolvimento 
cognitivo e social, e encontra aporte teórico nos estudos de Piaget, Vygotsky, PerretClermont, Dolle, Coll e outros. Por outro lado, o estudo da interação a partir de uma abordagem tecnológica, envolve a análise e proposição de modelos computacionais que possam refletir as trocas entre o sujeito e o ambiente computacional (Bassani, 2006). Este estudo foca o estudo da interação a partir de uma abordagem tecnológica, envolvendo a relação sujeito-meio. Entende-se que a percepção destas interações pode auxiliar no reconhecimento do perfil do sujeito/usuário, participante de um curso a distância.

$\mathrm{Na}$ especificação da relação sujeito-meio, o sujeito é facilmente identificado como o aluno, aqui entendido como sujeito-aluno. Entretanto, o meio pode ser entendido como um sistema de relações e "constitui um conjunto organizado em sistemas de elementos em interação" (Dolle, 1993). Dentre suas características, compreende, ao mesmo tempo, hierarquia, complexidade crescente e mobilidade hierárquica. Conforme Dolle (1993), em todo meio, seja ele qual for, encontram-se pessoas (sujeitos), objetos naturais e/ou artificiais e regras institucionais, regendo as relações interindividuais. A Figura 2 apresenta os elementos constitutivos do meio.

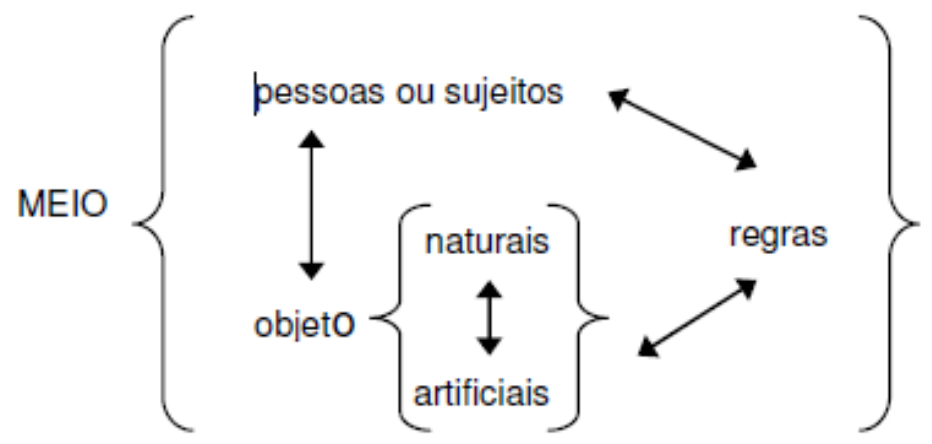

\section{Figura 2 - Elementos constitutivos do meio (Dolle, 1993)}

Cada sujeito, embora esteja em interação com a totalidade dos elementos do meio, conforme as inflexões de sua atividade, encontra-se mais em interação com as pessoas, os objetos ou as regras. Apesar de estar em interação com a totalidade dos objetos do meio, não o consegue, a cada instante, da mesma maneira.

MEIO

Neste estudo, entende-se que o material digital instrucional, disponibilizado em um curso na Web, caracteriza-se como um dos elementos constitutivos do meio, referenciado como objeto. Sendo assim, este trabalho foca as interações que se constituem essencialmente entre sujeito $\leftrightarrow$ material digital. Posteriormente, pretende-se ampliar este estudo de forma a contemplar, também, as interações interindividuais.

\section{CONSIDERAÇÕES FINAIS}

Seguindo as novas tendências das ferramentas de informação e comunicação da Web Semântica, os sistemas de ensino Web estão cada vez mais adaptados aos perfis dos usuários (Razmerita, 2003). Na personalização destes, as ontologias passam a ser cada vez mais utilizadas (Dolog, 2004a, 2004b, 2004c). Em consonância com a proposta da Web Semântica proposta por Berners-Lee et al. (2001), neste projeto de pesquisa propomos o desenvolvimento de uma ontologia que oriente a ação de agentes, de forma a contemplar as interações entre usuários e material instrucional digital, potencializando a usabilidade para usuários com necessidades especiais. Na modelização desta ontologia será utilizada a linguagem de ontologias OWL-DL, através da ferramenta de geração de ontologias Protégé. Tendo em vista a construção de um sistema que envolve agentes que trocam informações sobre as formas de interação dos usuários, pode-se fazer uso de uma camada semântica 
(Visser, 2000), que promova a comunicação entre os agentes, possibilitando a identificação de diferenças entre os diversos modos de interação dos usuários.

Generalizando, o objetivo desta ontologia é a de representar o conhecimento sobre interação em sistemas de aprendizagem Web, de forma a potencializar o acesso a material instrucional na Web, permitindo a adaptação desta a diferentes tipos de usuários, especialmente no que se refere a navegabilidade, interatividade e cognição.

Nesta perspectiva, entende-se que este trabalho amplia o escopo das pesquisas que vêm sendo desenvolvidas na área de inclusão, possibilitando, também, o acesso a usuários que apresentam necessidades especiais, através de conceitos agrupados de forma funcional, demonstrando como as formas de interação identificam o perfil dos usuários.

Assim, a ontologia trará no seu arcabouço uma conceitualização que servirá para identificar o tipo de necessidades especiais dos usuários do sistema de aprendizagem via Web. A próxima etapa deste estudo consiste em realizar a observação dos modos de interação dos usuários no uso de softwares educacionais, registrando-as e posteriormente coletando os conceitos que formarão o núcleo da ontologia.

\section{Referências Bibliográficas}

ALVARES, Luís Otávio Campos; SICHMAN, Jaime Simão (1997). Introdução aos Sistemas Multiagentes. In: XVI Jornada de Atualização em Informática. Brasilia/DF, Brasil.

BASSANI, Patrícia B. Scherer. Mapeamento das interações em ambiente virtual de aprendizagem: uma possibilidade para avaliação em educação a distância. Porto Alegre: PPGIE/UFRGS, 2006. Tese de Doutorado.

BERNERS-LEE, T., HENDLER, J. e LASSILA, O. The Semantic Web. In: Scientific American. Maio, 2001.

COSTA, Marcello Thiry Comicholi da. Uma Arquitetura Baseada em Agentes para Suporte ao Ensino a Distância. Tese de Doutorado. PPGEP, UFSC. Florianópolis/SC,

Brasil.

DOLLE, Jean-Marie. Para além de Freud e Piaget: referenciais para novas perspectivas em psicologia. Petrópolis, RJ: Vozes, 1993.

DOLOG, P.; HENZE, N.; NEJDL, W. e Sintek, M. Personalization in Distributed eLearning Environments. In Proc. of WWW2004 - The Thirteenth International World Wide Web Conference. Maio, 2004a.

DOLOG, P.; HENZE, N. e NEJDL, W. Reasoning and Ontologies for Personalized ELearning.To appear in Educational Technology \& Society, 2004b.

DOLOG, P., HENZE, N. NEJDL, W. e SINTEK, M. The Personal Reader: Personalizing and Enriching Learning Resources using Semantic Web Technologies. In Proc. of AH2004 - International Conference on Adaptive Hypermedia and Adaptive Web-Based Systems, August, 2004c, Eindhoven, The Netherlands. Springer Verlag.

FERREIRA, L. F. \& BERCHT, M. Agentes pedagógicos como apoio à avaliação de competência técnica em educação e prática médica em ambientes de realidade virtual.

In: XI SIMPÓSIO BRASILEIRO DE INFORMÁTICA NA EDUCAÇÃO. Maceió/Al, Brasil. Novembro, 2000.

GÓMEZ-PÉREZ, A., FERNÁNDEZ-LÓPEZ, M., CORCHO, O. Ontological engineering: With examples from the areas of knowledge management, ecommerce and the semantic web. Berlim, Springer-Verlag, 2004.

GRUBER, T. A Translation Approach to Portable Ontology Specifications. Knowledge Acquisition, 5(2), 199-220, 1993.

JENNINGS, N., SYCARA, K., WOOLDRIDGE M. A Roadmap of Agent Research and Development, 1998. 
LITWIN, Edith. Educação a Distância: temas para o debate de uma nova agenda educativa. Porto Alegre, Artmed, 2001.

MAEDCHE A.; STAAB S. Ontology Learning for the Semantic Web. IEEE Intelligent Systems, Special Issue on the Semantic Web, 2001, 16( 2).

NECHES, R.; FIKES, R. E.; FININ, T.; GRUBER, T R.; PATIL, R.; SENATOR, T. e Swartout, W. R. (1991). Enabling technology for knowledge sharing. AI Magazine, 12, 3556.

OWL (2004). OWL Web Ontology Language Guide < http://www.w3.org/ TR/2004/RECowl-guide-20040210/\# OwlVarieties> Accesso em: 02 abril. 2007.

NOVELLO, Taisa Carla (2003) "Ontologias, Sistemas baseados em Conhecimento e Modelos de Banco de Dados". Disponível em http://www.inf.ufrgs.br/clesio/cmp151/ cmp15120021/artigo_taisa.pdf. Acesso : 6/05/2003.

RAZMERITA, L. V. Modèle Utilisateur et Modélisation Utilisateur dans lês Systèmes de Gestion des Connaissances: une Approche fondée sur les Ontologies.

l'IRIT Toulouse, France, 2003. Tese de Doutorado.

SOWA, J., F. Knowledge Representation: Logical, Philosophical, and Computational. Foundations. Brooks Cole Publishing Co., Pacific Grove, CA, 2000.

VICCARI, Rosa Maria. Um tutor inteligente para a programação em Lógica Idealização, Projeto e Desenvolvimento. Coimbra, Portugal. Universidade de Coimbra, 1990. Tese de Doutorado.

ISSER, U.; STUCKENSCHMIDT, H.; VOGELE, T. Enabling techinologies for interoperability. In U. Visser and Pundt (EDs): Workshop on the 14th International Symposium of Computer Science for Environmental Protection, 2000, p. 35-46.

WOOLDRIDGE, M. (1997). Agent-Based Computing. Baltzer Journals, September 29, 1997 